\title{
Redistribution of thorotrast into a liver allograft several years following transplantation: a case report
}

\author{
Alyssa M Krasinskas ${ }^{1}$, Justina Minda ${ }^{1}$, Scott H Saul ${ }^{2}$, Abraham Shaked ${ }^{3}$ and Emma E Furth ${ }^{1}$ \\ ${ }^{1}$ Department of Pathology and Laboratory Medicine, University of Pennsylvania Medical Center, \\ Philadelphia, PA, USA; ${ }^{2}$ Department of Pathology, Chester County Hospital, West Chester, PA, USA and \\ ${ }^{3}$ Department of Surgery, University of Pennsylvania Medical Center, Philadelphia, PA, USA
}

\begin{abstract}
Thorotrast was used as a radiographic contrast agent in the United States from about 1930 to the mid-1950 s. Its use was discontinued when it was recognized that its radioactivity caused long-term deleterious effects. Such long-term sequelae of intravascular Thorotrast injection include, most notably, hepatic and hematologic malignancies and hepatic fibrosis. Some patients who had received Thorotrast subsequently received liver transplants. However, it was not known whether or not Thorotrast could become redistributed within the new allograft. A single report in 1994 demonstrated that radioactivity was detected by $\gamma$-ray spectroscopy in liver allografts shortly after transplantation. No report has identified Thorotrast in the allografts of long-term transplant survivors, and the redistribution of Thorotrast into allografts has not been documented histologically or by electron microscopy. We report a case of recurrent Thorotrast into a liver allograft 10 years posttransplant. We evaluated the native liver and allograft specimens for the presence of thorium utilizing light microscopy, electron microscopy and electron X-ray microanalysis. This case report demonstrates for the first time the redistribution of Thorotrast into a long-surviving liver allograft using histologic, electron microscopic and X-ray microanalysis techniques.
\end{abstract}

Modern Pathology (2004) 17, 117-120, advance online publication, 19 November 2003; doi:10.1038/modpathol.3800008

Keywords: Thorotrast; orthotopic liver transplantation; electron microscopy with X-ray microanalysis

Thorotrast, or thorium dioxide $\left({ }^{232} \mathrm{ThO}_{2}\right)$, was used as a radiographic contrast medium in the United States from approximately 1930 to 1954 . It was an ideal radiographic agent at the time because it had excellent clarity and it could be administered painlessly intravenously or intra-arterially. Thorotrast was used primarily for hepatosplenography, paranasal sinus visualization and angiographic examination of the head and limbs. However, it was an $\alpha$-particle emitter that resulted in long-term radioactive side effects. ${ }^{1}$ These side effects, including granuloma formation at the injection site, fatal blood dyscrasias and angiosarcomas, tended to occur 5 years to greater than 20 years following exposure. Owing to such long-term consequences, Thorotrast was eventually replaced by other nonradioactive contrast media.

Correspondence: EE Furth, Department of Pathology and Laboratory Medicine, 6 Founders, University of Pennsylvania Medical Center, 3400 Spruce Street, Philadelphia, PA 19104, USA.

E-mail: eef@mail.med.upenn.edu

Received 01 July 2003; revised 19 August 2003; accepted 06 October 2003; published online 19 November 2003
Thorotrast is initially deposited in all tissues of the human body and becomes redistributed within the reticuloendothelial system, particularly the liver $(60 \%)$, spleen $(30 \%)$ and bone marrow $(10 \%)$, over time. ${ }^{1,2}$ Orthotopic liver transplantation (OLT) has been performed for patients with Thorotrast exposure who develop Thorotrast-related malignancies or fibrosis isolated to the liver. ${ }^{3,4}$ Thorotrast redistributes within organs with time and tends to accumulate in fibrous tissue. ${ }^{1}$ Only one report has examined the redistribution of Thorotrast into liver allografts. ${ }^{4}$ The researchers in this study utilized $\gamma$-ray spectroscopy in a rat model and found that Thorotrast accumulated in the new allografts. They demonstrated that the Thorotrast in donor livers likely came from recipient spleen, bone marrow and lung. The same study also reported two patients with Thorotrast exposure who had evidence of Thorotrast in their liver allografts at 90 and 150 days post-transplant; histologic assessment of their allografts was not performed. ${ }^{4}$ In our report, we describe a patient who accumulated Thorotrast in his liver allograft, which was readily identifiable on routine 
hematoxylin and eosin sections 10 years following OLT.

\section{Case report}

A 57-year-old white male carpenter received a liver transplant for presumed primary sclerosing cholangitis. His past medical history was significant for episodes of variceal bleeds, ulcerative colitis and left knee exploratory surgery 36 years prior for a work-related injury; during the evaluation for his knee injury, he received Thorotrast contrast medium.

The patient's explanted liver weighed $1330 \mathrm{~g}$. Upon gross examination, the capsular and cut surfaces were nodular. The common hepatic duct and large intrahepatic ducts were dilated and contained granular stone debris. No distinct masses were noted. Histological sections of the liver showed irregular, dense, fibrous septae containing abundant intra- and extracellular deposits of coarse, brown, refractile, but nonbirefringent material, consistent with Thorotrast (Figure 1). Mild bile ductular proliferation, chronic inflammation, periseptal Mallory's hyaline and periportal cholestasis were also noted. The common hepatic duct showed periductal fibrosis and acute and chronic inflammation. No fibro-obliterative lesions, 'onion skinning' of the bile ducts or ductopenia were noted. The iron stain showed no hemosiderin deposition. Based on the histopathologic features, it was felt that the cirrhosis was most likely related to Thorotrast with a secondary contribution from chronic biliary obstruction; however, involvement of the large ducts by primary sclerosing cholangitis could not be excluded.

The patient did well until 10 years post-transplant when his liver enzyme values became elevated

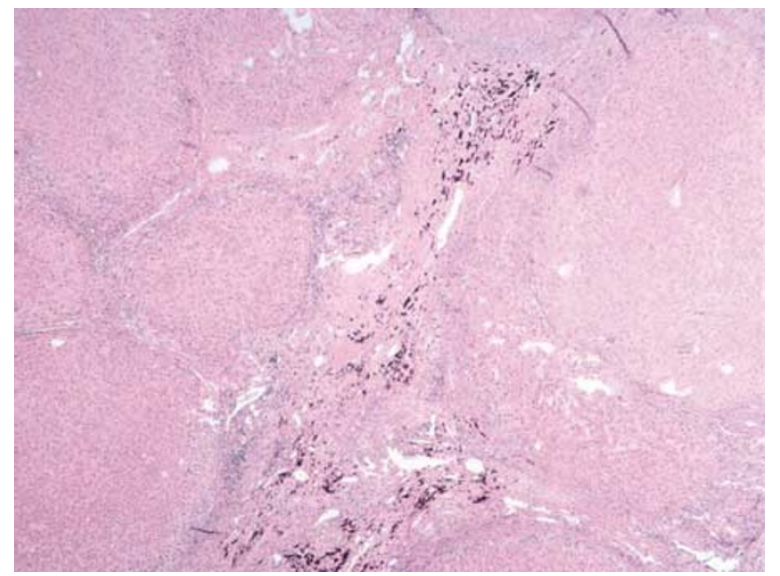

Figure 1 Histologic appearance of Thorotrast within the liver explant. In this representative fibrotic portal tract, Thorotrast appears as dense aggregates of dark-brown refractile material. Chronic inflammatory cells are focally present, but no significant interface activity was noted in multiple sections of the liver. The Thorotrast appeared to be located both within cells and scattered within the collagenous extracellular matrix (hematoxylin- and eosin- stained-sections, X25). (alanine aminotransferase $129 \mathrm{U} / \mathrm{l}$, aspartate aminotransferase $149 \mathrm{U} / \mathrm{l}$, total bilirubin $1.4 \mathrm{mg} / \mathrm{dl}$ ) and he developed mental status changes. A liver biopsy performed at this time showed mild acute cellular rejection without increased fibrosis. Rare, refractile, granular substance consistent with Thorotrast was also noted in this biopsy (Figure 2) and subsequent biopsies performed 1 month and 3 years later (this substance was not noted on earlier liver biopsies performed within the first 3 years of his transplant). The patient died 15 years following OLT from metastatic melanoma, which was first diagnosed 4 years prior in his left preauricular skin. Postmortem examination was not performed.

\section{Electron microscopy with X-ray microanalysis}

To confirm that Thorotrast had recurred in the liver allograft, electron microscopy with X-ray
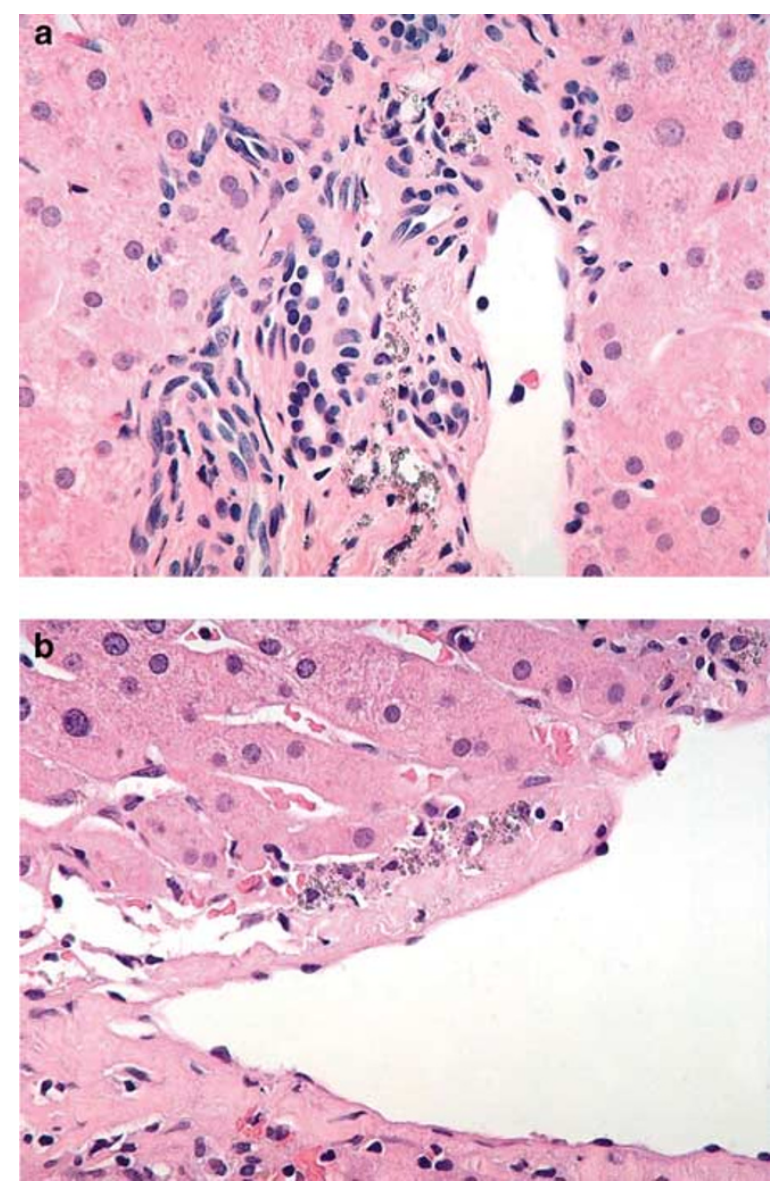

Figure 2 Histologic appearance of Thorotrast within a liver biopsy performed 10 years post-transplant. (a) Brown, refractile granules characteristic of Thorotrast are present within this portal tract; they appear to be located within mononuclear cells. (b) Collections of Thorotrast are also present within macrophages, and possibly extracellularly, around terminal hepatic venules/ central veins (hematoxylin- and eosin-stained sections, (a and b) $\mathrm{X} 400$ ). 


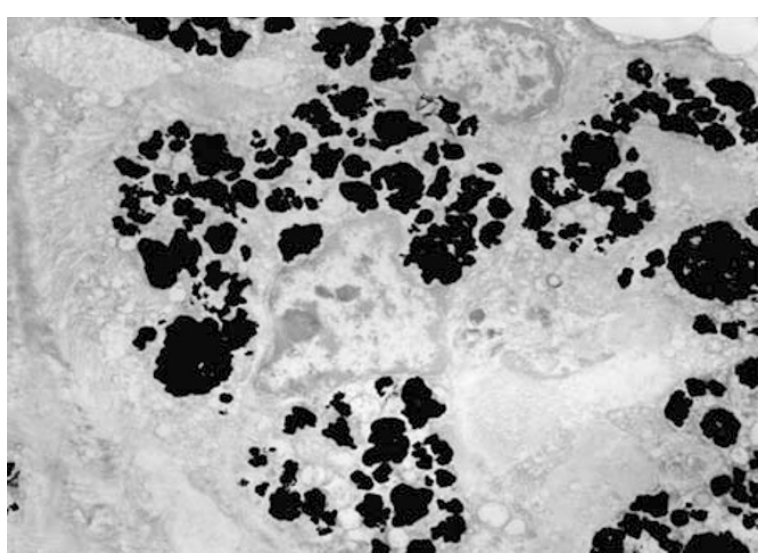

Figure 3 Electron microscopic appearance of Thorotrast. Electron microscopy performed on sections from the native liver shows numerous electron-dense deposits within the cytoplasm of a mononuclear cell; no definite membranes are seen around the deposits (glutaraldehyde fixation, X10,500). Similar electrondense deposits were present extracellularly within the collagen matrix and in tissue obtained from an allograft biopsy obtained 10 years post-transplant (not shown).

microanalysis was performed on samples from the liver explant as well as on sections from the biopsy of the allograft obtained 10 years after the transplant. Tissue grids were examined with a transmission electron microscope (Hitachi H-600, Nissei Sanyo Co) equipped with an energy dispersive X-ray elemental analysis system (Kevex System 8000) and the spectra for gold were analyzed. Electrondense deposits were seen within tissue samples from both the liver explant and the allograft biopsy (Figure 3). The X-ray spectrum from microanalysis of the electron-dense deposits showed a characteristic peak of thorium (M-line at 3.0) in both the liver explant (Figure 4a) and the allograft biopsy (Figure $4 \mathrm{~b})$. Other elements that were present included trace amounts of lead, iron, platinum, osmium, phosphorus, cobalt, chloride and sulfur.

\section{Discussion}

Although the use of Thorotrast as a radiographic contrast agent was discontinued by 1955 in the United States and most other countries, its longterm sequelae persist in recipients of the agent even to this day. Since Thorotrast is an $\alpha$-particle emitter and has an extremely long biological half-life, reportedly 400 years in humans, ${ }^{1}$ its accumulation in the body leads to chronic exposure and tissue injury. The most notorious long-term effects, often occurring more than 15 years following exposure, are liver and hematopoietic malignancies and liver fibrosis. In the largest recorded long-term study of patients who had received Thorotrast, the rate ratio for all deaths of Thorotrast patients started to increase more than 20 years following Thorotrast exposure. ${ }^{5}$ In addition, the rate ratios for liver cancer (hepatocellular carcinoma, cholangiocarcinoma and angiosarcoma), liver cirrhosis and leukemia were
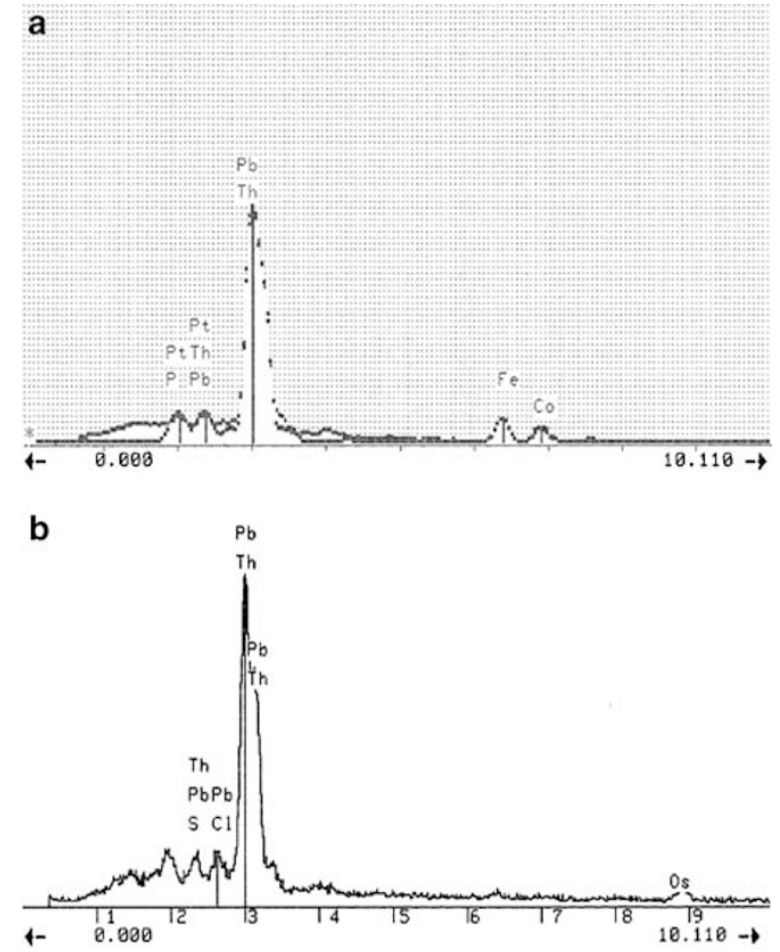

Figure 4 Electron microscopic X-ray microanalysis. Spectra obtained from analysis of the native liver explant (a) and a liver biopsy 10 years post-transplant (b) are shown. The X-axis represents $0-10 \mathrm{keV}$. The characteristic Thorotrast peak ( $\mathrm{M}$-line) at $3.0 \mathrm{keV}$ is seen in both specimens.

35.9, 6.9 and 12.5 times higher, respectively, than for controls. ${ }^{5}$

Our patient was exposed to Thorotrast in the $1950 \mathrm{~s}$ for radiographic evaluation of knee pain. Although the dose he received is not known, typical limb angiography involved injection of about $25 \mathrm{ml}$ of Thorotrast, which contained $5 \mathrm{~g}$ of thorium with an activity of $0.5 \mu \mathrm{Ci}{ }^{232} \mathrm{Th}$ and additional radioactivity from its progeny. ${ }^{6}$ Long-term effects of Thorotrast exposure have been reported with initial doses of as little as $14 \mathrm{ml}^{4,7}$ At 36 years following Thorotrast injection, our patient underwent OLT for end-stage liver disease and was diagnosed with Thorotrast-induced cirrhosis. At 10 years following OLT, Thorotrast was present in tissue biopsies of his allograft. Interestingly, this patient did not develop liver or hematopoietic malignancies associated with Thorotrast exposure..$^{2,5,6,8}$

Histologic examination of the patient's explanted liver revealed characteristic features of long-term Thorotrast deposition. ${ }^{7}$ With long-term exposure, the pattern of cirrhosis is often coarsely nodular. Aggregates of brown, refractile, nonbirefringent Thorotrast are present within cells and as extracellular deposits, located in areas of parenchymal fibrosis. There are often collections of the material in areas of subcapsular fibrosis. It has been reported that Thorotrast-induced portal tract fibrosis can cause biliary obstruction; $7^{7}$ however in our case, an 
exact etiology of the patient's biliary obstruction could not be determined.

Interestingly, histologic examination of the patient's liver allograft biopsies up to two and a half years post-transplant showed no evidence of Thorotrast deposition, while several biopsies taken 10 years post-transplant revealed Thorotrast deposition within portal macrophages and around the central veins (possibly extracellular) without associated fibrosis. This observation suggests that the redistribution and accumulation of Thorotrast into the patient's allograft to levels appreciable by light microscopy took several years, or may have been present only focally and missed on the early biopsies. Even at 10 years post-transplant, the pattern of deposition was different from that seen in the native explant, where the Thorotrast had 36 years to accumulate. These histologic findings are consistent with studies in the literature that examined Thorotrast accumulation in human livers over time. ${ }^{9}$ With a shorter time of exposure, the refractile Thorotrast material is often present in Kupffer cells located within sinusoids and uniformly distributed throughout the liver parenchyma from portal tract to central veins; it is not usually seen as extracellular deposits and fibrosis is not present. With time, aggregates of mononuclear cells containing the Thorotrast may form, and scant extracellular deposits may be seen. Eventually, the deposits become associated with areas of collagen deposition and form dense aggregates in areas of fibrosis, features characteristic of long-term Thorotrast accumulation.

To confirm that Thorotrast was present in the explant (native liver) and that it had recurred in the liver allograft, electron microscopy with X-ray microanalysis was performed. By electron microscopy, Thorotrast appears as dense granules ranging in size from 3 to $10 \mathrm{~nm}$, sometimes forming aggregates up to $2.8 \mu \mathrm{m}$ in size. ${ }^{9-11}$ When present within macrophages, the deposits may reside within the cytoplasm or within membrane-bound phagosomes, but they may be present within the collagenous extracellular matrix. ${ }^{9-12}$ An X-ray spectrum from electron microanalysis of Thorotrast deposits shows several characteristic peaks. The dominant peak is an M-line at about $3.0 \mathrm{keV}$, with additional peaks approximately as follows: $L \alpha=13.0 \mathrm{keV}, L \beta 2=$ $15.7 \mathrm{keV}, L \beta 1=16.2 \mathrm{keV}$ and $L \gamma=19.0 \mathrm{keV}^{10-12}$. Since the spectra used in our analyses extended only to $10 \mathrm{keV}$, only the prominent M-line peak at about $3.0 \mathrm{keV}$ was present. These electron microscopic analyses confirm the presence of Thorotrast in the patient's native liver and its recurrence in his liver allograft.
Although many patients exposed to Thorotrast may have received liver transplants over the years, only one report in the literature briefly mentioned the redistribution of Thorotrast into the liver allografts after a short post-transplant interval. ${ }^{4}$ Since the long-term survival of liver allograft recipients has improved dramatically over the past few years, and since our patient did not have a Thorotrast-induced malignancy, we were able to follow our patient for 15 years following transplant. During this prolonged post-transplant interval, we were able to document the presence of Thorotrast in his liver, an observation made at 10 years posttransplant. This case report demonstrates for the first time the redistribution of Thorotrast into a liver allograft using histologic, electron microscopic and $\mathrm{X}$-ray microanalysis techniques.

\section{References}

1 Strole WE, Wittenberg J. Case records of the Massachusetts General Hospital. Weekly clinicopathological exercises. Case 15-1981. N Engl J Med 1981;304:893-899.

2 Wegener $\mathrm{K}$, Wesch $\mathrm{H}$, Kampmann $\mathrm{H}$. Investigations into human thorotrastosis. Tissue concentrations of 232-Th and late effects in 13 autopsy cases. Virchows Arch A Pathol Anat Histol 1976;371:131-143.

3 Poggio JL, Nagorney DM, Nascimento AG, et al. Surgical treatment of adult primary hepatic sarcoma. Br J Surg 2000;87:1500-1505.

4 Spiethoff A, Wesch H, Wegener K, et al. Translocation of Thorotrast in the body. Radiat Res 1994;138:409-414.

5 Mori T, Kido C, Fukutomi K, et al. Summary of entire Japanese thorotrast follow-up study: updated 1998. Radiat Res 1999;152:S84-S87.

6 Mays CW. Alpha-particle-induced cancer in humans. Health Phys 1988;55:637-652.

7 da Horta JS. Late effects of thorotrast on the liver and spleen, and their efferent lymph nodes. Ann NY Acad Sci 1967;145:676-699.

8 Abbatt JD. History of the use and toxicity of thorotrast. Environ Res 1979;18:6-12.

9 Tessmer CF, Chang JP. Thorotrast localization by light and electron microscopy. Ann NY Acad Sci 1967;145:545-575.

10 Irie $\mathrm{H}$, Mori W. Long term effects of thorium dioxide (thorotrast) administration on human liver. Ultrastructural localization of thorium dioxide in human liver by analytical electron microscopy. Acta Pathol Jpn 1984;34:221-228.

11 Terzakis JA, Sommers SC, Snyder RW, et al. X-ray microanalysis of hepatic thorium depositions. Arch Pathol 1974;98:241-242.

12 Odegaard A, Ophus EM, Larsen AM. Identification of thorium dioxide in human liver cells by electron microscopic X-ray microanalysis. J Clin Pathol 1978;31:893-896. 